\title{
Prevalencia de adenomiosis en pacientes postoperadas de histerectomía con diagnóstico de sangrado uterino anormal
}

\author{
Prevalence of adenomyosis in postoperative hysterectomy \\ patients diagnosed with abnormal uterine bleeding
}

\author{
María de los Ángeles Chávez Loranca, * José Alan Arvizu Armenta, ${ }^{\ddagger}$ Luis Eligio Espinosa Hernández, \\ Pablo Mariano González Aldeco, ${ }^{\S}$ Alberto Woog Castañón, * Joana Carolina Juárez Tabares* \\ Citar como: Chávez LMÁ, Arvizu AJA, Espinosa HLE, González APM, Woog CA, Juárez TJC. Prevalencia \\ de adenomiosis en pacientes postoperadas de histerectomía con diagnóstico de sangrado uterino \\ anormal. Acta Med GA. 2022; 20 (1): 30-36. https://dx.doi.org/10.35366/103553
}

\section{Resumen}

Introducción: La adenomiosis es una entidad en la cual la prevalencia es muy amplia (5 a 70\%), con una media de 20 a $30 \%$. Actualmente, el estudio histopatológico de la pieza por histerectomía sigue siendo el único diagnóstico confirmatorio, así como la única manera de estudiar la prevalencia real. En nuestro medio, no se cuenta con muchos estudios actuales que nos digan la prevalencia de dicha patología, por lo cual es importante realizar búsquedas intencionadas. Objetivo: Estimar la prevalencia de adenomiosis en pacientes operadas de histerectomía total abdominal secundario a sangrado uterino anormal. Material y métodos: Estudio transversal, retrospectivo, descriptivo. Se realizó dicho estudio en pacientes postoperadas de histerectomía total abdominal por sangrado uterino anormal, cuyos reportes histopatológicos fueron recabados de enero 2017 a diciembre 2019. Se excluyeron pacientes con presencia de lesiones malignas (cáncer cervicouterino, cáncer de endometrio), que contaran con indicación de histerectomía por prolapso de órganos pélvicos, enfermedad pélvica inflamatoria, hemorragia obstétrica e histerectomía subtotal. Conclusiones: Se estudiaron en total 168 pacientes y se observó una prevalencia de $29.8 \%$, similar a lo establecido en la bibliografía en general. No se encontraron factores de riesgo asociados a dicho padecimiento.

Palabras clave: Adenomiosis, histerectomía, sangrado uterino anormal, miomatosis uterina, pólipos, estudio histopatológico.

\section{Abstract}

Introduction: Adenomyosis is an entity in which the prevalence is very wide ( 5 to $70 \%$ ), with a mean prevalence of 20 to $30 \%$. Currently, the only confirmatory diagnosis is the histopathological study of the piece by hysterectomy, as well as the only way to study the real prevalence. In our environment, there are not many current studies that tell us the prevalence of this pathology, which is why it is important to carry out intentional searches. Objective: To estimate the prevalence of adenomyosis in patients undergoing total abdominal hysterectomy secondary to abnormal uterine bleeding. Material and methods: Cross-sectional, retrospective, descriptive study. This cross-sectional, retrospective, descriptive study was carried out in patients who underwent total abdominal hysterectomy due to abnormal uterine bleeding, collecting their histopathological reports from January 2017 to December 2019, patients with the presence of malignant lesions (cervical cancer, endometrial cancer) were excluded, which had an indication for hysterectomy due to pelvic organ prolapse, pelvic inflammatory disease, obstetric hemorrhage, with subtotal hysterectomy. Conclusions: A total of 168 patients were studied, in which a prevalence of $29.8 \%$ was observed, similar to that established in the general bibliography. No risk factors associated with this condition were found.

Keywords: Adenomyosis, hysterectomy, abnormal uterine bleeding, uterine myomatosis, polyps, histopathological study.

\footnotetext{
* Residente de 4to año de Ginecología y Obstetricia. Alumna de la Facultad Mexicana de Medicina de la Universidad La Salle México.

* Médico de Ginecología y Obstetricia.

$\S$ Profesor Titular de Ginecología y Obstetricia.
}

\section{Correspondencia}

María de los Ángeles Chávez Loranca

Correo electrónico: angeleschl02@gmail.com

Aceptado: 31-05-2021. 


\section{INTRODUCCIÓN}

El sangrado uterino anormal es la segunda causa de consulta ginecológica, después de las infecciones cervicovaginales, siendo por ello una condición frecuente, con amplio impacto social. ${ }^{1,2}$ En México, de todas aquellas que lo padecen, anualmente sólo 6 millones buscan atención médica. ${ }^{3}$ En Estados Unidos se registran cerca de 600,000 histerectomías por este motivo. En el mundo existe una prevalencia de aproximadamente 3 a 30\% entre las mujeres en edad reproductiva. ${ }^{2}$

Por su origen puede ser de tipo anatómica o disfuncional. La Federación Internacional de Ginecología y Obstetricia (FIGO) cuenta con dos sistemas para el adecuado diagnóstico del sangrado uterino anormal: el sistema 1 es una revisión de la terminología y definiciones de los síntomas; mientras que el sistema 2 trata las causas y contribuciones al sangrado en el periodo reproductivo.

El sistema de clasificación FIGO 2 se divide en nueve categorías; están organizadas en el acrónimo PALM-COEIN ("palm-koin") (Figura 1). ${ }^{4}$

Las causas anatómicas benignas son tres:

1. Leiomiomatosis. Los tumores benignos del tracto genital femenino más comunes son los miomas uterinos. ${ }^{5}$ Se estima que $20-30 \%$ de las mujeres en edad reproductiva se ven afectadas por esta enfermedad y aumenta a $70 \%$ en las de 49 años. ${ }^{6}$

2. Pólipos. Es una enfermedad ginecológica común y una causa frecuente de sangrado uterino anormal entre las mujeres fértiles. Se estima que la prevalencia es de alrededor de 7.8 a $34.9 \%$. Las mujeres con pólipos endometriales generalmente experimentan sangrado uterino anormal en $68 \%$ de todos los casos, y $39 \%$ de
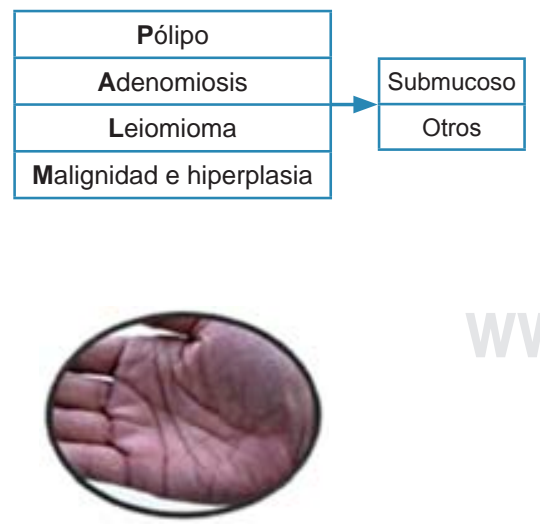
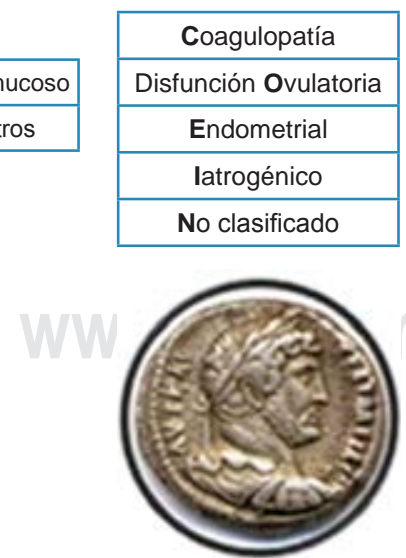

Figura 1: Sistema de clasificación PALM-COEIN. Modificada de: Munro MG et al. ${ }^{2}$ las premenopáusicas con sangrado uterino anormal tienen pólipos endometriales. ${ }^{6}$

3. Adenomiosis. El útero consta de tres capas de interior a exterior: endometrio, miometrio y serosa. El diagnóstico de adenomiosis se realiza cuando las glándulas endometriales y el estroma están presentes dentro de la musculatura del útero. ${ }^{7}$ La adenomiosis se diagnostica con mayor frecuencia en mujeres durante los años 40 y 50, lo que está en consonancia con la práctica clínica, donde las histerectomías son comunes en este grupo de edad. ${ }^{8}$ La adenomiosis es diagnosticada en la histología, algunas bibliografías han encontrado que el aumento de la paridad, la menarquia temprana $(\leq$ 10 años de edad), los ciclos menstruales cortos $(\leq 24$ días de duración), el índice de masa corporal (IMC) elevado y el uso de anticonceptivos orales fueron hallazgos estadísticamente significativos en pacientes con adenomiosis, lo que sugiere una asociación entre adenomiosis y exposición a estrógenos. ${ }^{9}$

La prevalencia reportada de adenomiosis varía ampliamente de 5 a $70 \%$, dependiendo del método utilizado para el diagnóstico, con una prevalencia media de 20 a $30 \% .{ }^{6}$ Se informa que la incidencia de adenomiosis ocurre en 20 a $65 \%$ de las mujeres y se asocia con el aumento de la edad. ${ }^{10}$ En un estudio realizado en población mexicana, la prevalencia de adenomiosis en piezas de histerectomía fue de $33.33 \%$ (86 de 258 pacientes). ${ }^{11}$

Aunque no se conoce la patogénesis de la adenomiosis, existen al menos cuatro teorías propuestas. La primera, la más comúnmente citada, se basa en el concepto de una lesión de tejido desregulada y mecanismo de reparación que promueve la migración celular y la invaginación del endometrio basal al miometrio. Esta desregulación es exacerbada por un estado hiperestrogénico y resistente a la progesterona, por lo tanto, fomenta la proliferación endometrial anormal y actividad uterina mediada por oxitocina elevada. ${ }^{12}$ Una segunda teoría es que la adenomiosis resulta de restos embrionarios de células pluripotentes de Müller fuera de lugar. Otras dos teorías, que no son tan usuales, son que la adenomiosis es causada por la invaginación de la membrana basal a lo largo del sistema linfático intramiometrial o que la adenomiosis es el resultado de las células madre de la médula ósea. ${ }^{7}$

Exploración física: los síntomas clínicos importantes incluyen sangrado uterino abundante (65\%) con dismenorrea (15-30\%) en promedio de ambas (40 a $60 \%$ ) o dolor pélvico crónico, aunque hasta $30 \%$ de las mujeres son asintomáticas. ${ }^{10} \mathrm{El}$ inicio de los síntomas se informa típicamente en mujeres entre las edades de 40 y 50. La infertilidad se encuentra en $11-12 \%$ de las pacientes. ${ }^{7}$ 


\begin{tabular}{l} 
Características ecográficas \\
Globular \\
Engrosamiento asimétrico \\
Quistes \\
Islas hiperecoicas \\
Sombreado en forma de \\
abanico \\
\hline
\end{tabular}

El agrandamiento uterino difuso se describe con frecuencia como un útero globular y es un hallazgo común en el examen físico cuando un paciente tiene adenomiosis. La adenomiosis también puede estar presente en la cavidad endometrial como una masa polipoide o puede formar adenomas, que son áreas focales de agregados nodulares circunscritos de músculo liso. Otras anormalidades uterinas asociadas son comunes en mujeres con adenomiosis como leiomiomas $(50 \%)$, endometriosis $(11 \%)$ y pólipos endometriales (7\%).

El diagnóstico de sospecha se puede realizar por ultrasonido o por resonancia magnética, éstos pueden revelar un aumento del volumen uterino, un miometrio engrosado, quistes de miometrio o una mayor heterogeneidad miometrial con pérdida de una unión clara de miometrio endometrial que son muy sugestivos de este trastorno. ${ }^{10}$ El ultrasonido se usa comúnmente como la modalidad de imagen inicial en la evaluación del dolor pélvico y clínicamente sospechado de adenomiosis. La sensibilidad y especificidad reportadas del ultrasonido transvaginal para el diagnóstico de adenomiosis son 53 a $89 \%$ y 67 a 98\%, respectivamente. Muchos casos de sospecha por ultrasonido se confirman con resonancia magnética, generalmente considerado el criterio de modalidad de imagen estándar con sensibilidad $78-88 \%$ y especificidad $67-93 \% .{ }^{13}$ Como observamos, ambos métodos de estudio tienen sensibilidad y especificidad similares.

Ultrasonido (USG) endovaginal: se han visto muchas características en escala de grises que se describen como representativas de la adenomiosis, por ejemplo: 1) las
Características ecográficas Ilustración

Líneas y vesículas subendometriales ecogénicas

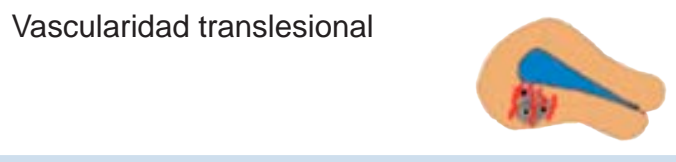

Zona de unión irregular

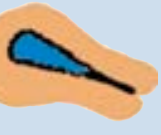

Zona de unión interrumpida

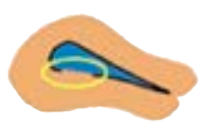

Figura 2: Características de la adenomiosis por ultrasonido transvaginal.

Modificada de: Tan J.12

características del borde endometrial (zona de unión), el hallazgo primario consiste en estrías ecogénicas y/o nódulos que se extienden desde el endometrio hacia el miometrio interno. 2) Los quistes miometriales en el contexto de adenomiosis son típicamente de 1 a $5 \mathrm{~mm}$ y son más comúnmente anecoicos. 3) El engrosamiento miometrial puede ser focal o difuso, aunque las áreas focales tienen bordes relativamente indistintos en comparación con los de los leiomiomas. Dependiendo de la extensión del proceso, el útero puede permanecer de tamaño normal, demostrar engrosamiento miometrial asimétrico o desarrollar agrandamiento globular difuso. 4) Sombras ciegas venecianas: es la ecotextura alterada del miometrio, que se vuelve heterogénea y gruesa, con sombras verticales delgadas, lo que resulta en una apariencia ciega veneciana. 5) La vascularización miometrial generalmente aumenta debido a la hiperplasia muscular e hipertrofia.

El patrón Doppler color de la adenomiosis consiste en un mayor número de vasos tortuosos que penetran en todo el miometrio involucrado, una apariencia que también se ve en la angiografía. Por lo tanto, el Doppler color es útil para distinguir la adenomiosis focal de los leiomiomas, que tienden a desplazar los vasos y demostrar el flujo circunferencial. $^{6}$

En la Figura 2 se muestran de manera ilustrada todos los signos de los que hemos hablado.

Resonancia magnética: es el segundo estudio o el de extensión en caso de que el ultrasonido no sea concluyente. Varios criterios de diagnóstico por resonancia magnética, tanto directos como indirectos, han sido descritos con 
base en la presencia de glándulas endometriales (adeno) dentro del miometrio e hipertrofia de células musculares lisas (miosis). Sin embargo, estos criterios no deben usarse a menos que el radiólogo sea un experto en conocimiento de la anatomía uterina por resonancia magnética. ${ }^{14}$ Se realizan en T2, la presencia de adenomiosis en la resonancia magnética generalmente se define como una medición de zona de unión mayor de 12 mm, y los hallazgos equívocos son representados por zonas de unión que miden entre 8 y $12 \mathrm{~mm}^{12}$

Algunos autores denominan a la adenomiosis como endometriosis interna, y reservan el término adenomiosis externa para el cuadro comúnmente llamado endometriosis. En la resonancia magnética se ha usado una clasificación de la adenomiosis (Figura 3).

La adenomiosis externa expuesta en esta clasificación, se refiere a aquella que surge en la parte externa del útero, lo que interrumpe la serosa, pero no afecta a la zona de unión. Siempre está asociada con la endometriosis profunda que parece ser el progenitor de este subtipo de adenomiosis. No obstante, desde un punto de vista clínico, se requiere consenso para adoptar una clasificación de adenomiosis con el objetivo no sólo de mejorar la reproducibilidad, sino también la predicción de la respuesta terapéutica y el pronóstico. ${ }^{14}$

Se puede obtener un diagnóstico histológico de adenomiosis a partir de biopsias miometriales histeroscópicas, sin embargo, se puede pasar por alto si la adenomiosis es más profunda que las muestras de biopsia tomadas o se encuentra en sitios que no se biopsian, se han reportado una sensibilidad subóptima y especificidad de 54.32 y $78.46 \%$, respectivamente. ${ }^{12}$

Ahora bien, el diagnóstico confirmatorio requiere hasta estos momentos un espécimen de histerectomía, para

\section{Interna}

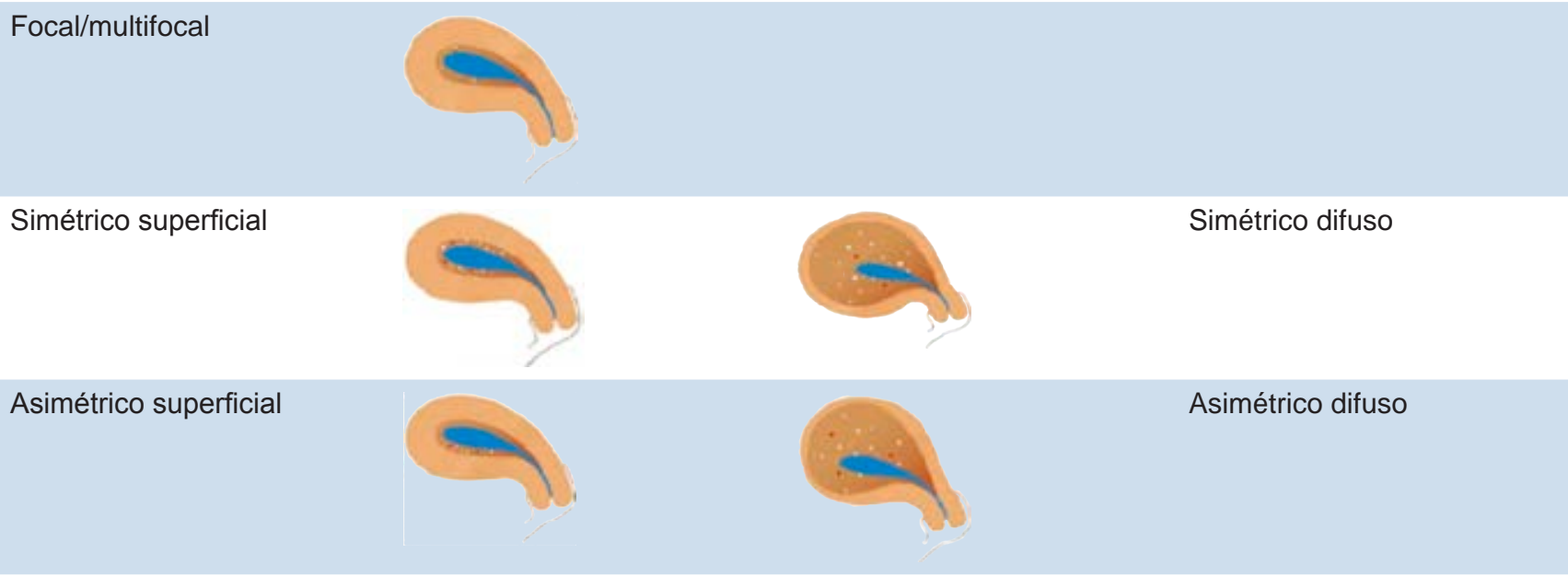

\section{Externo}

Posterior
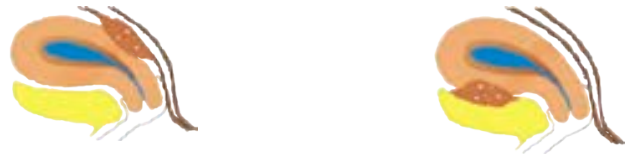

Anterior

\section{Adenomioma}

Sólido Sústico

Figura 3: Clasificación de la adenomiosis por resonancia magnética. Modificada de: Tan J. ${ }^{12}$ 
Tabla 1: Prevalencia de adenomiosis en pacientes con sangrado uterino anormal postoperadas de histerectomía ( $\mathrm{N}=168)$.
Tipo de prevalencia

$\mathrm{n}(\%)$

Adenomiosis

Leiomiomatosis

Pólipos

Endometriosis
$50(29.8)$

$144(85.7)$

$20(11.9)$

$7(4.2)$ así poder estudiar la totalidad de la pieza, al observarse la distancia mínima requerida para un diagnóstico se ha debatido, pero oscila entre la mitad y dos campos de baja potencia desde la unión endomiometrial o una profundidad mínima de invasión que varía de 1 a 4 mm. La implicación de al menos $25 \%$ a un tercio del grosor miometrial es otro criterio para el diagnóstico que se ha utilizado.

La histerectomía es el único tratamiento definitivo para la adenomiosis, el cual nos permite obtener a su vez el resultado de patología y, con ello, el diagnóstico definitivo.

Objetivo: estimar la prevalencia de adenomiosis en pacientes operadas de histerectomía total abdominal secundario a sangrado uterino anormal. Objetivos específicos: 1) conocer los grupos de edad con mayor frecuencia de adenomiosis. 2) identificar las comorbilidades presentes en las pacientes con diagnóstico de adenomiosis. 3) identificar paridad de pacientes con diagnóstico de adenomiosis. 4) conocer antecedente de uso de anticonceptivos en pacientes diagnóstico de adenomiosis.

\section{MATERIAL Y MÉTODOS}

Estudio transversal, retrospectivo, descriptivo.

Criterios de inclusión: pacientes postoperadas de histerectomía total abdominal por sangrado uterino anormal. Criterios de exclusión: 1) presencia de lesiones malignas (cáncer cervicouterino, cáncer de endometrio). 2) contar con indicación de histerectomía por prolapso de órganos pélvicos, enfermedad pélvica inflamatoria, hemorragia obstétrica. 3) histerectomía subtotal. Criterios de eliminación. No contar con la información completa.

Cálculo del tamaño de la muestra: se realizó un muestreo del total de pacientes con histerectomía como muestra a conveniencia.

Metodología para la recolección de la muestra: se recabaron reportes histopatológicos de los últimos tres años: enero 2017- diciembre 2019, cotejando con la base de datos obtenida de los censos del Servicio de Ginecología donde se recabarán las pacientes y sus datos postopera- torios de histerectomía con sangrado uterino anormal y reportes obtenidos en patología de esta institución. Separando a las pacientes con diagnósticos de adenomiosis, previa autorización del comité de bioética en investigación institucional.

Variables: edad, indicación de histerectomía, resultados histopatológicos, aumento de paridad, menarquia temprana, ciclos menstruales cortos, índice de masa corporal elevado, uso de anticonceptivos orales.

Las variables demográficas (edad e índice de masa corporal) se describieron utilizando una media y desviación estándar. Las variables demográficas (índice de masa corporal) ginecoobstétricas (menarca, paridad, ciclos menstruales, uso de anticonceptivos, indicación de histerectomía total abdominal) se describieron mediante frecuencias absolutas y relativas. El análisis de los datos se realizó utilizando SPSS versión 21.0 y considerando un valor de significancia estadística de $\mathrm{p}<0.05$.

\begin{tabular}{|c|c|c|c|c|}
\hline \multirow[b]{2}{*}{ Variable } & & \multicolumn{2}{|c|}{ Adenomiosis } & \multirow[b]{2}{*}{$p$} \\
\hline & & Sí & No & \\
\hline Cirugía uterina previa & $\begin{array}{l}\text { Sí } \\
\text { No }\end{array}$ & $\begin{array}{l}38 \\
12\end{array}$ & $\begin{array}{l}75 \\
43\end{array}$ & 0.116 \\
\hline $\begin{array}{l}\text { Salpingoclasia } \\
\text { bilateral }\end{array}$ & $\begin{array}{l}\text { Sí } \\
\text { No }\end{array}$ & $\begin{array}{l}19 \\
31\end{array}$ & $\begin{array}{l}41 \\
77\end{array}$ & 0.687 \\
\hline Alcoholismo & $\begin{array}{l}\text { Sí } \\
\text { No }\end{array}$ & $\begin{array}{l}14 \\
36\end{array}$ & $\begin{array}{l}24 \\
93\end{array}$ & 0.291 \\
\hline Tabaquismo & $\begin{array}{l}\text { Sí } \\
\text { No }\end{array}$ & $\begin{array}{l}13 \\
37\end{array}$ & $\begin{array}{l}29 \\
89\end{array}$ & 0.846 \\
\hline Dismenorrea & $\begin{array}{l}\text { Sí } \\
\text { No }\end{array}$ & $\begin{array}{l}27 \\
23\end{array}$ & $\begin{array}{l}62 \\
55\end{array}$ & 0.905 \\
\hline Hipertensión arterial & $\begin{array}{l}\text { Sí } \\
\text { No }\end{array}$ & $\begin{array}{r}7 \\
43\end{array}$ & $\begin{array}{l}23 \\
95\end{array}$ & 0.425 \\
\hline Diabetes mellitus & $\begin{array}{l}\text { Sí } \\
\text { No }\end{array}$ & $\begin{array}{r}7 \\
43\end{array}$ & $\begin{array}{r}7 \\
111\end{array}$ & 0.084 \\
\hline Menarca & $\begin{array}{l}\text { Sí } \\
\text { No }\end{array}$ & $\begin{array}{r}6 \\
44\end{array}$ & $\begin{array}{r}17 \\
101\end{array}$ & 0.678 \\
\hline $\begin{array}{l}\text { Uso de } \\
\text { anticonceptivos orales } \\
\text { combinados }\end{array}$ & $\begin{array}{l}\text { Sí } \\
\text { No }\end{array}$ & $\begin{array}{r}6 \\
44\end{array}$ & $\begin{array}{l}20 \\
98\end{array}$ & 0.417 \\
\hline $\begin{array}{l}\text { Índice de masa } \\
\text { corporal }\end{array}$ & $\begin{array}{l}\text { Normal } \\
\text { Sobrepeso } \\
\text { Obesidad }\end{array}$ & $\begin{array}{r}9 \\
24 \\
17\end{array}$ & $\begin{array}{l}24 \\
55 \\
37\end{array}$ & 0.915 \\
\hline Gestas & $\begin{array}{l}\text { No multigesta } \\
\text { Multigesta }\end{array}$ & $\begin{array}{l}27 \\
23\end{array}$ & $\begin{array}{l}75 \\
43\end{array}$ & 0.246 \\
\hline Edad & $\begin{array}{l}\text { Premenopausia } \\
\text { Postmenopausia }\end{array}$ & $\begin{array}{r}43 \\
7\end{array}$ & $\begin{array}{r}101 \\
17\end{array}$ & 0.945 \\
\hline
\end{tabular}


Tabla 3: Características demográficas.

\begin{tabular}{|c|c|c|}
\hline & \multicolumn{2}{|c|}{ Media (DE) } \\
\hline & Sí & No \\
\hline Edad (años) & $47(6)$ & $46(7)$ \\
\hline IMC $\left(\mathrm{kg} / \mathrm{m}^{2}\right)$ & 44.48 (114.44) & $28.31(4.36)$ \\
\hline Peso (kg) & $70.44(10.59)$ & 70.66 (11.13) \\
\hline Talla (m) & $1.57(0.06)$ & $1.58(0.06)$ \\
\hline Peso de útero $(\mathrm{g})$ & 231.10 (163.78) & $290.67(318.63)$ \\
\hline
\end{tabular}

\section{RESULTADOS}

Se estudiaron 168 pacientes en el periodo de tres años. En la Tabla 1 se observa el porcentaje de prevalencia de las diferentes patologías, observándose $29.8 \%$ en la adenomiosis, similar a la registrada en la bibliografía.

En la Tabla 2 se buscaron factores de riesgo asociados para presentar dicha patología (adenomiosis), Los factores buscados fueron cirugía uterina previa, salpingoclasia bilateral, alcoholismo, tabaquismo, dismenorrea, hipertensión arterial, diabetes mellitus, menarca temprana, uso de anticonceptivos orales, IMC, número de gestas y edad (pre-y postmenopáusicas); sin embargo, no se encontró ninguno donde la $p<0.05$, por lo que concluimos que no hubo significancia estadística.

En la Tabla 3 se observó que el IMC tenía una media de 44.48, con mayor relevancia en pacientes con IMC elevado, y en cuanto a la edad, una media de 47 , inclinándose más hacia la etapa postmenopáusica, pero esto podría estar sesgado por la edad en que se realizan las histerectomías y no propiamente con la patología.

No se pudo realizar comparativo entre diagnóstico por USG previo versus postoperadas por falta de estudios complementarios en los expedientes. De esta manera, podemos observar cómo en la población mexicana no hay variación en la prevalencia media de la adenomiosis, siendo hasta una tercera parte de las causantes del sangrado uterino anormal, de allí la importancia de poder mejorar el método de sospecha diagnóstica por medios de imagen, y así realizar más diagnósticos, previo a la resolución quirúrgica de la misma.

\section{DISCUSIÓN}

Según la bibliografía comentada en este artículo, se ha reportado una prevalencia muy amplia, 5 a 70\%, depen- diendo del método utilizado para el diagnóstico, con una prevalencia media de 20 a 30\%. ${ }^{6}$ En este caso se realizó el estudio con el diagnóstico definitivo que es histopatológico y se encontró similitud en la prevalencia media reportada, y de un estudio en población mexicana de $33.33 \%$, los resultados fueron de $29.8 \%$ en nuestras pacientes. ${ }^{11}$

Se estudiaron las variables como factores de riesgo: uso de anticonceptivos, paridad aumentada, menarca temprana o ciclos cortos, ${ }^{9}$ sin encontrarse una verdadera significancia estadística, se agregaron otros probables factores de riesgo como enfermedades crónico-degenerativas o contar con salpingoclasia, y de la misma manera se descartaron como factores asociados. Observamos una media en el índice de masa corporal elevado. ${ }^{9}$ Vemos cómo coincide que la adenomiosis aumente con la edad por la media resultante, ${ }^{10}$ sin embargo, esto podría estar sesgado por el grupo de edad que se realizan las histerectomías. ${ }^{8}$

Se necesitarían más pacientes para realizar una muestra más grande y observar si los factores de riesgo se modifican, y en el diagnóstico podría cotejarse si se contara con todos los estudios de USG previa cirugía para comparar cuáles coinciden con el diagnóstico patológico, contando con el protocolo completo para realizar un nuevo estudio de esta patología.

\section{CONCLUSIÓN}

Se estudiaron en total 168 pacientes, en el cual se observó una prevalencia de $29.8 \%$, similar a lo establecido en la bibliografía en general. No se encontraron factores de riesgo asociados a dicho padecimiento.

\section{REFERENCIAS}

1. Munro MG, Critchley HOD, Fraser IS; FIGO Menstrual Disorders Committee. The two FIGO systems for normal and abnormal uterine bleeding symptoms and classification of causes of abnormal uterine bleeding in the reproductive years: 2018 revisions. Int J Gynaecol Obstet. 2018; 143 (3): 393-408.

2. Munro MG, Critchley HO, Broder MS, Fraser IS; FIGO Working Group on Menstrual Disorders. FIGO classification system (PALM-COEIN) for causes of abnormal uterine bleeding in nongravid women of reproductive age. Int J Gynaecol Obstet. 2011; 113 (1): 3-13.

3. Diagnóstico y tratamiento del sangrado uterino anormal de origen no anatómico. México: Instituto Mexicano del Seguro Social; 2015.

4. ACOG committee opinion no. 557: Management of acute abnormal uterine bleeding in nonpregnant reproductive-aged women. Obstet Gynecol. 2013; 121 (4): 891-896.

5. Benetti-Pinto CL, Rosa-E-Silva ACJS, Yela DA, Soares Júnior JM. Abnormal uterine bleeding. Rev Bras Ginecol Obstet. 2017; 39 (7): 358-368.

6. Cunningham RK, Horrow MM, Smith RJ, Springer J. Adenomyosis: a sonographic diagnosis. Radiographics. 2018; 38 (5): 1576-1589.

7. Struble J, Reid S, Bedaiwy MA. Adenomyosis: a clinical review of a challenging gynecologic condition. J Minim Invasive Gynecol. 2016; 23 (2): 164-185. 
8. Abbott JA. Adenomyosis and abnormal uterine bleeding (AUB-A)pathogenesis, diagnosis, and management. Best Pract Res Clin Obstet Gynaecol. 2017; 40: 68-81.

9. Nakayama $\mathrm{H}$, Teramoto $\mathrm{H}$, Teramoto $\mathrm{M}$. True incidence of uterine adenomatoid tumors. Biomed Rep. 2013; 1 (3): 352-354.

10. Bacon JL. Abnormal uterine bleeding: current classification and clinical management. Obstet Gynecol Clin North Am. 2017; 44 (2): 179-193.

11. Arellano PEI, Labastida TJ. Prevalencia de adenomiosis en piezas quirúrgicas de histerectomía y factores de riesgo clínicos relacionados. Acta Med. 2018; 16 (1): 15-22.

12. Tan J, Yong P, Bedaiwy MA. A critical review of recent advances in the diagnosis, classification, and management of uterine adenomyosis. Curr Opin Obstet Gynecol. 2019; 31 (4): 212-221.
13. Konrad J, Merck D, Wu JY, Tuomi A, Beland M. Improving ultrasound detection of uterine adenomyosis through computational texture analysis. Ultrasound Q. 2018; 34 (1): 29-31.

14. Bazot $M$, Darai E. Role of transvaginal sonography and magnetic resonance imaging in the diagnosis of uterine adenomyosis. Fertil Steril. 2018; 109 (3): 389-397.

Conflicto de intereses: Los autores de este artículo declaran que no tienen conflicto de intereses.

Todos los procedimientos seguidos fueron de acuerdo con los estándares éticos del Comité de Bioética del Nuevo Sanatorio Durango y con la Declaración de Helsinki de 1975, revisada en 2000. 